
intervention for perinatal care in Nepal (NePeriQIP); study protocol of a cluster randomised trial

Ashish KC, ${ }^{1,2}$ Anna Bergström, ${ }^{1,3}$ Dipak Chaulagain, ${ }^{1,4}$ Olivia Brunell, ${ }^{1}$ Uwe Ewald, ${ }^{1}$ Abhishek Gurung, ${ }^{4}$ Leif Eriksson, ${ }^{5}$ Helena Litorp, ${ }^{1}$ Johan Wrammert, ${ }^{1}$ Erik Grönqvist, ${ }^{6}$ Per-Anders Edin, ${ }^{7}$ Claire Le Grange, ${ }^{1}$ Bikash Lamichhane, ${ }^{8}$ Parashuram Shrestha, ${ }^{8}$ Amrit Pokharel, ${ }^{8}$ Asha Pun, ${ }^{2}$ Chahana Singh, ${ }^{2}$ Mats Målqvist ${ }^{1}$

To cite: KC A, Bergström A, Chaulagain $\mathrm{D}$, et al. Scaling up quality improvement intervention for perinatal care in Nepal (NePeriQIP); study protocol of a cluster randomised trial. BMJ Glob Health

2017;2:e000497. doi:10.1136/ bmjgh-2017-000497

Handling editor Seye Abimbola

Received 26 July 2017

Revised 15 September 2017

Accepted 18 September 2017

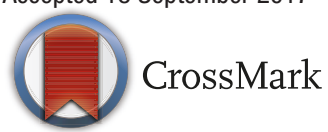

${ }^{1}$ International Maternal and Child Health, Department of Women's and Children's Health, Uppsala University, Uppsala, Sweden

${ }^{2}$ Health Section, UNICEF, UN House, Lalitpur, Nepal ${ }^{3}$ Institute for Global Health, University College London, London, UK

${ }^{4}$ Lifeline Nepal, Kathmandu, Nepal

${ }^{5}$ Department of Public Health and Caring Sciences, Uppsala University, Uppsala, Sweden

${ }^{6}$ Health Economic Forum, Uppsala University, Uppsala, Sweden

${ }^{7}$ Department of Economics, Uppsala University, Uppsala, Sweden

${ }^{8}$ Department of Health Services, Ministry of Health, Nepal

Correspondence to Dr Mats Målqvist;

mats.malqvist@kbh.uu.se

\section{ABSTRACT}

Introduction Nepal Perinatal Quality Improvement Project (NePeriQIP) intends to scale up a quality improvement (QI) intervention for perinatal care according to WHO/National guidelines in hospitals of Nepal using the existing health system structures. The intervention builds on previous research on the implementation of Helping Babies Breathequality improvement cycle in a tertiary healthcare setting in Nepal. The objective of this study is to evaluate the effect of this scaled-up intervention on perinatal health outcomes.

Methods/design Cluster-randomised controlled trial using a stepped wedged design with 3 months delay between wedges will be conducted in 12 public hospitals with a total annual delivery rate of 60000 . Each wedge will consist of 3 hospitals. Impact will be evaluated on intrapartum-related mortality (primary outcome), overall neonatal mortality and morbidity and health worker's performance on neonatal care (secondary outcomes). A process evaluation and a cost-effectiveness analysis will be performed to understand the functionality of the intervention and to further guide health system investments will also be performed.

Discussion In contexts where resources are limited, there is a need to find scalable and sustainable implementation strategies for improved care delivery. The proposed study will add to the scarce evidence base on how to scale up interventions within existing health systems. If successful, the NePeriQIP model can provide a replicable solution in similar settings where support and investment from the health system is poor, and national governments have made a global pledge to reduce perinatal mortality. Trial registration number ISRCTN30829654.

\section{INTRODUCTION}

Stillbirths and neonatal deaths account for $>5.6$ million deaths annually and have been observed as a huge global health challenge. ${ }^{1}$ Over two-thirds of these deaths are preventable and take place in countries and in settings

\section{Key messages}

What is already known about this topic?

- There is a growing interest for the need to strengthen quality of care globally.

- Helping Babies Breathe has been proven effective in reducing intrapartum stillbirths and early neonatal mortality.

- Quality improvement initiatives have the potential to promote sustainability of intervention effects.

What are the new findings?

- There is a dearth of evidence on how to take quality of care interventions to scale in weak health systems.

- The proposed study will supply evidence on health outcomes after scaling up of quality improvement measures within a public health system in a low-resource setting.

- Insights and experiences of the process of strengthening the health system to deliver better quality of care will be documented and provided.

What are the recommendations for policy and practice?

- Improving quality of care during the perinatal period should be a prioritised area for investment.

- Efforts to scale up evidence-based interventions for improved quality of care need to be investigated.

where resources are scarce. ${ }^{12}$ These deaths are the reflection of huge societal inequities and inaccessibility to quality care at and around the time of birth. ${ }^{3}$ With the transition from Millennium Development Goals to the new global Sustainable Development Goals, absolute targets have been set to reduce stillbirth and neonatal mortality. ${ }^{4}$ In the last 25 years, investment has been made to identify cost-effective interventions such as intrapartum 
fetal heart rate surveillance, neonatal resuscitation, early initiation of breastfeeding, Kangaroo Mother Care (KMC), infection prevention and management to reduce mortality during the intrapartum and neonatal period. ${ }^{5-7}$ However, translation of these evidence-based interventions into effective clinical practice and at scale has been a challenge in low resource settings where the economic benefit is high. ${ }^{389}$ In an effort to provide guidance for implementation of perinatal interventions to countries, WHO has recommended Standards for improving quality of maternal and newborn care in health facilities. ${ }^{10}$ However, these proposed standards for care require rigorous evaluation in health system context in terms of its ability to translate science into practice.

The research group has previously conducted a study on a quality improvement (QI) model for standard neonatal resuscitation (Helping Babies Breathe-quality improvement cycle (HBB-QIC)) in a tertiary hospital setting in Nepal and reported improvements in health worker's performance and perinatal outcome. ${ }^{11}{ }^{12}$ However, the upscale of this model in the health system setting has yet to be evaluated in terms of its effectiveness and sustainability.

There were two major lesson learnt from this previous study evaluating HBB-QIC. First, effort on neonatal resuscitation reduced intrapartum stillbirth and first-day mortality. However, it did not reduce overall perinatal mortality, ${ }^{13}$ so a comprehensive neonatal care intervention is needed to see overall change in perinatal mortality. Therefore, the Nepal Perinatal Quality Improvement Project (NePeriQIP) focuses on the continuum of care during the perinatal period, adding training on all aspects of the National Neonatal Clinical Protocol to the HBB training.

Second, a sustainable approach for QI implementation is necessary especially in settings where the resources for support are scare and inconsistent. Systematic reviews have also shown that a combination of implementation strategies, such as weekly review meetings, and periodic simulated skill checks, checklists and self-evaluation is a more effective strategy to improve the clinical performance than a single strategy. ${ }^{14-18}$ This was also the experience from the previous HBB-QIC trial, where multiple implementation strategies were used. Process audit and feedback complemented training, which resulted in retention of skills learnt during training and new practices implemented in the clinic. ${ }^{19}$

Nepal has pledged to reduce the current stillbirth rate of 18 per 1000 births to 13 and newborn mortality to 11 per 1000 live births by 2035 , committing to the Every Newborn Action Plan. ${ }^{20}{ }^{21}$ Improving quality of care around the time of birth will save lives and reduce morbidity but require a functional health system. Similar to other low-income countries, delivering quality health services to the population remains a challenge in Nepal, despite increase in number of human resources and improved infrastructure. ${ }^{22}{ }^{23}$ With the nationwide introduction of demand side financing for improving access to maternal and neonatal care in the health facilities since 2005 , the number of women delivering in institutions has increased by threefold. ${ }^{23-25}$

An assessment of quality of perinatal care in hospitals of Nepal has shown that health workers do not perform as per the standards and lack necessary tools and environment to implement those standards. ${ }^{26}$ The context of rapid increase in institutional deliveries and poor quality of care warrants improvement in the structures, systems and performance for perinatal care.

In response to these needs and with the lessons learnt from previous trials, NePeriQIP intends to scale up an extended version of the HBB-QIC package for improved perinatal care in hospitals in Nepal. Primary objective of this study is to evaluate impact of this scaled-up intervention on intrapartum mortality (intrapartum stillbirth and first-day mortality). Secondary objectives are to assess the effect of the intervention model on overall neonatal mortality and morbidity and health worker's performance on neonatal care, and to evaluate the process of implementation. A cost-effectiveness analysis to further guide health system investments will also be performed.

\section{METHODS \\ Setting}

The NePeriQIP intervention will be implemented in 12 district level public hospitals in Nepal. Hospitals with $>1000$ deliveries per year were identified. The selected hospitals had an annual delivery rate ranging from approximately 1000 to 11000 , and an estimated intrapartum mortality rate of $20 / 1000$ births (table 1 ). The geographical location of these hospitals are scattered all throughout the country, mostly in the flat lands. These hospitals are the referral hospitals for providing maternal and perinatal care. We selected these hospitals with the criteria of having government health facilities with deliveries $>1000$ per year.

\section{Intervention}

The extended HBB-QIC will be administered to all 12 hospitals. NePeriQIP will be rolled out in three phases: preparatory, implementation and sustainability phase.

\section{Preparatory phase}

In order to enable the introduction and implementation of NePeriQIP, mentors will be recruited externally by central Ministry of Health. These mentors will be clinicians with experience of working in perinatal health. They will be responsible to revitalise and orient the administration at each hospital to implement the NePeriQIP intervention. Using set selection criteria 2-4, QI facilitators will be recruited internally at each hospital. These facilitators will be responsible to implement the QI interventions in clinical units in each of the 12 hospitals. The QI facilitators and mentors will be trained on facilitation skills and standard perinatal care package by the research team. As a first task the QI facilitators will together with clinical unit staffs conduct assessment of 
Table 1 Estimated numbers of deliveries at included hospitals and the possible change in primary outcome possible to detect at each hospital (alfa 0.05, beta 0.8).

\begin{tabular}{|c|c|c|c|c|c|}
\hline & $\begin{array}{l}\text { Wedge } 1 \\
\text { ( } 3 \text { months baseline) }\end{array}$ & $\begin{array}{l}\text { Deliveries } \\
2015\end{array}$ & $\begin{array}{l}\text { Estimated number } \\
\text { of deliveries before } \\
\text { intervention }\end{array}$ & $\begin{array}{l}\text { Estimated number } \\
\text { of deliveries after } \\
\text { intervention } \\
\text { (9 months follow-up) }\end{array}$ & $\begin{array}{l}\text { Change in intrapartum } \\
\text { mortality possible to } \\
\text { detect given baseline } \\
20 / 1000\end{array}$ \\
\hline 1 & Western Regional Hospital & 9427 & 2400 & 9400 & $8 / 1000$ \\
\hline 2 & $\begin{array}{l}\text { Mid-Western Regional } \\
\text { Hospital }\end{array}$ & 3139 & 750 & 2400 & $13 / 1000$ \\
\hline 3 & Bardiya District Hospital & 1065 & 250 & 800 & N/A \\
\hline
\end{tabular}

\begin{tabular}{|c|c|c|c|c|c|}
\hline & $\begin{array}{l}\text { Wedge } 2 \\
\text { ( } 6 \text { months baseline) }\end{array}$ & & & & \\
\hline 4 & Bharatpur Hospital & 11318 & 5700 & 8500 & $7 / 1000$ \\
\hline 5 & Seti Zonal Hospital & 5767 & 2900 & 3900 & $9 / 1000$ \\
\hline \multirow[t]{2}{*}{6} & Nuwakot District Hospital & 1438 & 700 & 1100 & $16 / 1000$ \\
\hline & $\begin{array}{l}\text { Wedge } 3 \\
\text { ( } 9 \text { months baseline) }\end{array}$ & & & & \\
\hline 7 & Koshi Zonal Hospital & 8355 & 6300 & 6300 & $7 / 1000$ \\
\hline 8 & $\begin{array}{l}\text { Rapti Sub-Regional } \\
\text { Hospital }\end{array}$ & 3280 & 2500 & 2500 & $10 / 1000$ \\
\hline 9 & $\begin{array}{l}\text { Nawalparasi District } \\
\text { Hospital }\end{array}$ & 1374 & 1000 & 1000 & $15 / 1000$ \\
\hline
\end{tabular}

\begin{tabular}{|c|c|c|c|c|c|}
\hline & $\begin{array}{l}\text { Wedge } 4 \\
\text { (12 months baseline) }\end{array}$ & & & & \\
\hline 10 & Lumbini Zonal Hospital & 9007 & 9000 & 6800 & $6 / 1000$ \\
\hline 11 & Bheri Zonal Hospital & 4276 & 4300 & 3200 & $8 / 1000$ \\
\hline \multirow[t]{2}{*}{12} & Pyuthan District Hospital & 1194 & 1200 & 900 & $15 / 1000$ \\
\hline & Total & 60571 & 37000 & 46800 & \\
\hline
\end{tabular}

$\mathrm{N} / \mathrm{A}$, not available.

the readiness, availability and quality of perinatal care. Based on the findings, a causal/bottleneck analysis on inadequacy of quality of perinatal care will be conducted. Results will be shared with the hospital administration and a plan will be developed and implemented to improve the quality of perinatal care. Routine use of inpatient sick newborn register will be strengthened to improve the monitoring of perinatal care. This phase will take 3 months for completion.

\section{Implementation phase}

The implementation phase will commence with series of trainings to build capacity of health workers on WHO Standards for Improving Quality of Maternal and Newborn Care in health facilities ${ }^{10}$ and the National Neonatal Clinical Protocol. ${ }^{27}$ This training will build on the HBB methodology and be extended to comprehensively cover delivery and essential newborn care. After 6 months of implementation, refresher training will be done to health workers on clinical standards.
During the implementation phase, the mentors will support and supervise the QI facilitators on a periodical basis and conduct individual in-house training if deemed necessary. The implementation phase will be ongoing for 9 months.

\section{Sustainability phase}

In order to fully implement and sustain the NePeriQIP intervention, the QI facilitators will be expected to continue with QI activities as an integral component of daily practice.

\section{Study design}

A stepped-wedge cluster-randomised design will be applied and the hospitals will be randomly allocated to four wedges with different time points for initiation of intervention; each wedge will thus include three hospitals (figure 1). ${ }^{28}$ The delay in intervention start will be 3 months, meaning that the preparatory phase will be completed for each step before starting the next. Randomisation was performed proportionate to number of 


\begin{tabular}{|c|c|c|c|c|c|c|c|c|c|c|c|c|c|c|c|c|c|c|c|c|c|c|c|c|}
\hline \multirow[b]{2}{*}{ Hospital } & \multicolumn{24}{|c|}{ Months } \\
\hline & 1 & 2 & 3 & 4 & 5 & 6 & 7 & 8 & 9 & 10 & 11 & 12 & 13 & 14 & 15 & 16 & 17 & 18 & 19 & 20 & 21 & 22 & 23 & 24 \\
\hline \multicolumn{25}{|l|}{1} \\
\hline \multicolumn{25}{|l|}{2} \\
\hline \multicolumn{25}{|l|}{3} \\
\hline \multicolumn{25}{|l|}{4} \\
\hline \multicolumn{25}{|l|}{5} \\
\hline \multicolumn{25}{|l|}{6} \\
\hline \multicolumn{25}{|l|}{7} \\
\hline \multicolumn{25}{|l|}{8} \\
\hline \multicolumn{25}{|l|}{9} \\
\hline \multicolumn{25}{|l|}{10} \\
\hline \multicolumn{25}{|l|}{11} \\
\hline \multicolumn{25}{|l|}{12} \\
\hline & & & & & & & & & & Ro & utine & data & col & ecti & ons & & & & & & & & & \\
\hline & & Bas & line & & & & & & & $\mathrm{Imp}$ & leme & ntati & & & & & & Sust & taina & bility & & & & \\
\hline
\end{tabular}

Figure 1 Gantt chart for intervention design.

deliveries per year in each hospital starting by allocating one of the four largest hospitals to each wedge and then continued with the middle-range hospitals. Finally, the four hospitals with the least deliveries per year were randomly allocated to each wedge. This implies that all hospitals will eventually receive the intervention with a total duration of 12 months (3 months preparatory phase and 9 months implementation phase). In order to achieve a sustained effect of the intervention, the hospital managements will be stimulated to continue beyond the intervention period of 12 months.

At the hospitals with most expected deliveries in each wedge on-location observations of clinical practice during delivery and immediate postnatal care will be performed.

All three phases will be evaluated through process evaluation where documentation of activities and decisions, trainings, activities and the supportive supervision of mentors will be gathered. Qualitative data on perceptions, attitudes and understanding of the process will be collected through focus group discussions (FGDs), in-depth interviews (IDIs) and ethnographic data collection methods. Further detail of process evaluation will be published elsewhere.

A cost-effectiveness analysis will be performed after completion of intervention, calculating cost of lives saved and disability-adjusted life years (DALYs) averted and quality-adjusted life years (QALYs) gained.

\section{Data collection}

Data collection will start at all hospitals at the same time (figure 1) and continue until all hospitals have completed the full intervention period of 12 months (preparatory and Implementation phases) and at least 3 months of sustainability phase. Thus, data collection will be ongoing at all hospitals for a total of 24 months (figure 1). To standardise data collection process, the research group will use the WHO Standards for Improving Quality of
Maternal and Newborn Care in health facilities. ${ }^{10}$ A team of data collectors, supervised by a research manager, will be established to collect information on mortality outcomes, health worker's performance, beneficiaries' perception and process documentation (table 2). The data collectors will extract information from the records of the hospital, conduct exit client interviews and observe clinical practices on perinatal care in hospitals. A standardised data collection protocol will be used to ensure consistency and stringency at the different hospitals. Training will be provided to the data collectors on selection criteria of women, taking consent, clinical observation, interview and data retrieval. Pregnant women with gestational age $\geq 22$ weeks with a fetal heart sound at admission who agree to participate in the study will be eligible for the study.

\section{Registry data}

A uniform data collection system will be established in the 12 hospitals. Data collection will be separate from QI implementation and will start at the same time in all 12 hospitals. Data collectors provided by the research project will work in close collaboration with the Medical Record Officer at each hospital to ensure quality of routine documentation. Data will be extracted from the medical records and registries for the full study period.

\section{Exit interviews}

Exit interviews will be conducted by the team of data collectors with all mothers on discharge from all study hospitals during the study period. A semi-structured questionnaire will be used in order to capture maternal recall of delivery care and descriptive background factors. Any women who meet the eligibility criteria will be informed about the study and requested to participate and the data collector will take the informed written consent from women agreeing to participate in the study. 
Table 2 Outcome measures

\begin{tabular}{|c|c|c|}
\hline & Indicator & Study design \\
\hline \multirow[t]{2}{*}{$\begin{array}{l}\text { Primary outcome } \\
\text { measures }\end{array}$} & $\begin{array}{l}\text { Intrapartum mortality measured as intrapartum } \\
\text { stillbirth (death within uterus } \geq 22 \text { weeks of gestation } \\
\text { or birth weight } \geq 500 \mathrm{~g} \text { ) and }\end{array}$ & Registry data \\
\hline & $\begin{array}{l}\text { First-day neonatal mortality (deaths within first } 24 \\
\text { hours of birth) }\end{array}$ & Registry data \\
\hline Secondary outcome & Early (0-6 days) and late neonatal (7-27 days) in- & Registry data \\
\hline
\end{tabular}

measures

hospital mortality

Admittance to Sick Newborn Care Units and

Sick Newborn Surveillance and Registry data

morbidity epidemiology

Rate and severity of neonatal encephalopathy

Sick Newborn Surveillance

Health workers' performance:

- on fetal surveillance in clinical settings

- on neonatal resuscitation in simulated and

Clinical Observations, Registry data and Exit interviews clinical settings

- on essential newborn care (immediate newborn care, cord care practices, breast feeding, Kangaroo Mother Care, routine assessment of newborn)

- on infection prevention and management

Beneficiaries' satisfaction on the received care

Exit interviews

Acceptability and adequacy of each implementation Focus Group Discussions and In-Depth Interviews strategy component

Perceptions and attitudes of perinatal care practices Focus Group Discussions and In-Depth Interviews among health workers and families

Cost-effectiveness of the intervention

Research administration records

\section{Sick newborn surveillance}

All newborns admitted to the Sick Newborn Care Unit or paediatric wards will be registered using a separate follow-up form. Data on reason for admission and medications given will be noted.

\section{Clinical observations}

Clinical observations will be conducted to capture the clinical variables extracted from the WHO standards. Tools for observation of labour management and postnatal care ${ }^{29}$ will be developed and piloted.

\section{Social autopsy}

In order to assess the social and demographic factors associated with stillbirth and neonatal death, a social autopsy tool will be used to interview the relative of the deceased baby at the time of discharge. This tool, which has been validated in similar settings and widely used, ${ }^{30}$ will help to identify what needs to be improved at home, community and health facility level to reduce the delay in care of mother and newborn.

Knowledge and skill evaluation

Knowledge and skill evaluation will be done at four different time points to collect information on the health workers competency on intrapartum fetal surveillance, neonatal resuscitation, immediate newborn care, breast feeding, KMC, infection prevention and management.
Economic evaluation

Cost-effectiveness of the quality improvement intervention will be compared with usual care from the societal perspective during the trial period. The comparator will be standard treatment at district level public hospitals in Nepal prior to the NePeriQIP intervention. The cost per saved life, incremental cost-effectiveness ratio per QALY gained and DALY averted will be calculated under the assumption that the NePeriQIP intervention does not affect morbidity and quality of life. This assumption is similar to assumption made by Vossius et $a l^{31}$

The cost-effectiveness will also be calculated under different scenarios of how the intervention affects quality of life, where data on morbidity (eg, birth weight, cognitive function, neonatal encephalopathy) collected at discharge (and follow-up) will be used to assess the likelihood of different scenarios.

The evaluation has a societal perspective. All costs for healthcare at delivery, and all costs related to the implementation and sustainability of the intervention will be recorded. The latter includes costs for training (eg, costs for instructors and time lost for healthcare professionals under training), costs related to changed practice and costs for maintaining the QI intervention (eg, time cost for follow-up and retraining). Secondary data sources on healthcare costs, income and labour market outcomes (Nepal Annual Household Survey) 
and life expectancy in the general population will be used.

\section{Sample size and power calculation}

Power calculation for the primary outcome is based on preliminary estimates of an intrapartum mortality of 20/1000 births and 60000 deliveries in the study hospitals per year. We estimated the intraclass correlation coefficient to 0.01 , based on previous findings from literature. ${ }^{32} 33$ A 1 year sample according to the stepped-wedge design with 12 clusters (hospitals) would preliminarily allow us to demonstrate a significant reduction of intrapartum mortality of $14 / 1000$ births, that is, to $6 / 1000$ or less (alfa 0.05 , beta 0.80 ). Calculations performed with $\mathrm{R}$ package CRTsize.

Given the limitation of the cluster-randomised design to detect changes in small proportion we have also calculated the change in intrapartum mortality possible to detect at each hospital when comparing baseline (variable number of months) and intervention period (9 months) (table 1).

\section{Data management}

For the purpose of ensuring high-quality data collection, management and data security an independent data monitoring committee will be formed. All data will be saved on a server and backed up on a weekly basis. The project manager and the data manager will make spot checks monthly at the 12 hospitals to verify records with the primary source of data. A quality control team from Uppsala University will provide oversight to ensure quality of data collection and to avoid data loss on a regular basis. Procedures for data storage and handling will follow strict ethical review board and confidentiality rules.

\section{Data analysis}

Analysis of the intervention package effectiveness will be based on comparison of intervention and control group according to the stepped-wedge randomised design and a before-after design at each hospital. Descriptive analyses and logistic regression will be used to detect differences between study arms and time points. Cross-sectional data gathered through the established surveillance system and clinical observations will produce a large data set allowing for epidemiological analyses. Qualitative data from FDGs and IDIs will be analysed by thematic analysis and framework analysis. The final report of this protocol will follow the general Consolidated Standards of Reporting Trials Statement.

\section{Ethical consideration}

All research involving newborn infants needs careful ethical consideration, mainly since the subjects themselves cannot agree to whether they want to participate in the study or not. Based on our previous experience from studies in a tertiary hospital of Nepal, there is no adverse effect of the implementation of standard perinatal protocol. ${ }^{11}$ By using the stepped-wedge design all hospitals will eventually be included and receive the intervention.

Clinical observations will monitor the performance of health staff. The data collectors will not make reminders to healthcare staff if failing to follow protocol, unless there is an imminent threat to the life of mother or child. Withholding knowledge must be weighed towards the benefit of the study. Harmful practices must however be corrected immediately.

Written informed consent will be obtained before collection through face-to-face methods like exit interviews, FGDs or IDIs. All ethical principles outlined in the World Medical Association (WMA) Declaration of Helsinki will be observed.

\section{Methodological limitations}

There are certain limitations to the study. First, since some of these hospitals are geographically located in adjacent districts, there is a risk of contamination of intervention roll out as there are routine periodic meetings of the health workers of different hospitals. However, since the intervention is not only consisting of new knowledge being taught but also includes structural changes at the hospital after intervention the contamination will be limited. Second, the information on the stillbirths and neonatal death is from the record registers, there is a chance of reporting bias (differential misclassification) by the health workers. Third, since there will be a clinical observation by surveillance officer in each hospital throughout the study period, there is a risk of observer's bias in recording and registry practices, as well as a strengthening effect on clinical practice that might dilute results. Close monitoring of the data collection process will be implemented, with onsite supervision and continuous capacity-building of data collectors to curb the potential problems with reporting and observation biases.

\section{DISCUSSION}

Different QI initiatives for perinatal health have been piloted and proposed in small-scale models in different countries, including Nepal. However, these QI interventions have not been rigorously evaluated in district health system contexts. We propose a scaled-up intervention to put structures, systems and processes in place for quality improvement for perinatal care in hospital settings that is scalable and sustainable. A close collaboration with the Ministry of Health and the existing structures within the health system are essential components to get the needed buy-in and to secure sustainability of the initiative. Multiple interactions with the Ministry of Health have been undertaken as part of developing the implementation guidelines, which if proven successful might be disseminated and scaled up to all hospitals in the country. An implementation guideline development workshop was held in Kathmandu in March 2017 to get 
input from important stakeholders and to mainstream the initiative.

The Global Strategy for Women, Children and Adolescent's Health 2016-2030 paves the road for the health-related sustainable development goals by focusing on disability-free survival. ${ }^{34}$ However, evidence on the effects of neonatal interventions on morbidity and impairment is sparsely available from low-income and middle-income settings. Data on neonatal morbidity such as neonatal encephalopathy and impairment following intrapartum hypoxia are not available from the hospitals in low-income setting like Nepal. As a result, good estimates of country-specific burden of disease information is not available.

The global Every Newborn Action Plan launched by UN's secretary general in 2014 World Health Assembly highlights a need for global attention for perinatal health. The plan urged countries to make quality of care for mothers and newborn a priority as well as strengthen the data for better accountability. NePeriQIP proposes a multifaceted strategy for how quality of perinatal care can be improved in a health system setting and aims to generate evidence for better perinatal care outcomes.

Acknowledgements The study group would like to thank Professor Susan Niermeyer, University of Colorado, USA, Professor Stefan Swartling Peterson, Chief Health Section, Programme Division, UNICEF Head Quarter New York, Dr Rajendra Prasad Pant, Director General, Department of Health Services Government of Nepal who reviewed and provided input for the design and data collection components of the study.

Contributors $\mathrm{AK}$ and MM conceptualised the study and AB, UE, DC, OB and JW contributed to the development of study design. AG, HL, PE, EG, LE and CL made additions and changes to data collection development. AK and MM drafted the firs draft of the manuscript. All authors read and approved of the final version.

Funding Einhorn Foundation, Swedish Foundation for International Cooperation in Research and Higher Education (STINT), Swedish Research Council (VR), Laerdal Foundation for Acute Medicine, Norway and UNICEF Nepal country office will support the funding for the study.

Competing interests None declared.

Ethics approval Ethical review group of Nepal Health Research Council (Ref 20171643, 16 March 2017).

Provenance and peer review Not commissioned; externally peer reviewed.

Open Access This is an Open Access article distributed in accordance with the Creative Commons Attribution Non Commercial (CC BY-NC 4.0) license, which permits others to distribute, remix, adapt, build upon this work non-commercially, and license their derivative works on different terms, provided the original work is properly cited and the use is non-commercial. See: http://creativecommons.org/ licenses/by-nc/4.0/

C) Article author(s) (or their employer(s) unless otherwise stated in the text of the article) 2017. All rights reserved. No commercial use is permitted unless otherwise expressly granted.

\section{REFERENCES}

1. Lawn JE, Blencowe H, Oza S, et al. Every Newborn: progress, priorities, and potential beyond survival. Lancet 2014;384:189-205.

2. Dickson KE, Simen-Kapeu A, Kinney MV, et al. Every Newborn: health-systems bottlenecks and strategies to accelerate scale-up in countries. Lancet 2014;384:438-54.

3. Darmstadt GL, Kinney MV, Chopra M, et al. Who has been caring for the baby? Lancet 2014;384:174-88.

4. Affairs UNDoEaS. United Nations General Assembly- Transforming our world: the 2030 Agenda for Sustainable Development. New York: United Nations Department of Economic and Social Affairs, 2015.
5. Bhutta ZA, Das JK, Bahl R, et al. Can available interventions end preventable deaths in mothers, newborn babies, and stillbirths, and at what cost? Lancet 2014;384:347-70.

6. Moran AC, Kerber K, Sitrin D, et al. Measuring coverage in $\mathrm{MNCH}$ : indicators for global tracking of newborn care. PLoS Med 2013;10:e1001415.

7. Bhutta ZA, Yakoob MY, Lawn JE, et al Stillbirths: what difference can we make and at what cost? Lancet 2011;377:1523-38.

8. Darmstadt GL, Oot DA, Lawn JE. Newborn survival: changing the trajectory over the next decade. Health Policy Plan 2012;27 (Suppl 3):iii1-5.

9. Kc A, Rana N, Målqvist $\mathrm{M}$, et al. Effects of delayed umbilical cord clamping vs early clamping on Anemia in Infants at 8 and 12 months: a randomized clinical trial. JAMA Pediatr 2017;171:264.

10. World Health Organisation (WHO). Standards for improving quality of maternal and newborn care in health facilities. Geneva: WHO Press, 2016.

11. Kc A, Wrammert J, Clark RB, et al. Reducing perinatal mortality in Nepal using helping babies breathe. Pediatrics 2016;137:e20150117.

12. Wrammert J, Sapkota S, Baral K, et al. Teamwork among midwives during neonatal resuscitation at a maternity hospital in Nepal. Women Birth 2017;30:262-9.

13. Wrammert J, Kc A, Ewald U, et al. Improved postnatal care is needed to maintain gains in neonatal survival after the implementation of the helping babies breathe initiative. Acta Paediatr 2017:106:1280-5.

14. Forsetlund L, Bjorndal A, Rashidian A, et al. Continuing education meetings and workshops: effects on professional practice and health care outcomes. The Cochrane database of systematic reviews 2009;2:CD003030.

15. Pageler NM, Longhurst CA, Wood M, et al. Use of electronic medical record-enhanced checklist and electronic dashboard to decrease CLABSIs. Pediatrics 2014;133:e738-46.

16. Davis DA, Taylor-Vaisey A. Translating guidelines into practice. A systematic review of theoretic concepts, practical experience and research evidence in the adoption of clinical practice guidelines. CMAJ 1997;157:408-16.

17. Lion $\mathrm{KC}$, Ebel $\mathrm{BE}$, Rafton $\mathrm{S}$, et al. Evaluation of a quality improvement intervention to increase use of telephonic interpretation. Pediatrics 2015;135:e709-16.

18. Ambroggio L, Thomson J, Murtagh Kurowski E, et al. Quality improvement methods increase appropriate antibiotic prescribing for childhood pneumonia. Pediatrics 2013;131:e1623-31.

19. Kc A, Wrammert J, Nelin V, et al. Evaluation of Helping Babies Breathe Quality Improvement Cycle (HBB-QIC) on retention of neonatal resuscitation skills six months after training in Nepal. BMC Pediatr 2017;17:103.

20. World Health Organization, United Nations Children's Fund.In: Every Newborn: an action plan to end preventable deaths. Geneva: World Health Organization, 2014

21. Health Mo. In: Nepal's Every Newborn Action Plan 2015-2035. Kathmandu, Nepal: Ministry of Health, 2016.

22. Government of Nepal MoHaP. In: ed. National Health Policy 2071 Kathmandu, Nepal: Ministry of Health and Population, 2014.

23. Population MoHa. The Aama programme: an initiative towards reducing maternal and newborn deaths in Nepal. Kathmandu, Nepal: Family Health Division, 2010.

24. Ministry of Health and Population, New Era, ICF Macro. In: ed. Nepal Demographic and Health Survey 2006. Kathmandu: New Era, 2006.

25. Målqvist $\mathrm{M}$, Pun $\mathrm{A}, \mathrm{Kc} \mathrm{A}$. Essential newborn care after home delivery in Nepal. Scand J Public Health 2017;45:202-7.

26. UNICEF MoHaPG. Assessment of Readiness, Availability and Quality of Neonatal Services in 12. Kathmandu: Hospitals of Nepal, 2014.

27. Government of Nepal - Ministry of Health -Department of Health Services. National Neonatal Clinical Protocol: Teku, 2016.

28. Hemming K, Haines TP, Chilton PJ, et al. The stepped wedge cluster randomised trial: rationale, design, analysis, and reporting. $B M J$ 2015;350:h391.

29. Tripathi V, Stanton C, Strobino D, et al. Development and validation of an index to measure the quality of facility-based labor and delivery care processes in sub-saharan Africa. PLoS One 2015;10:e0129491.

30. Kalter HD, Salgado R, Babille M, et al. Social autopsy for maternal and child deaths: a comprehensive literature review to examine the concept and the development of the method. Popul Health Metr 2011:9:45

31. Vossius C, Lotto E, Lyanga S, et al. Cost-effectiveness of the "helping babies breathe" program in a missionary hospital in rural Tanzania. PLOS One 2014:9:e102080.

32. Campbell MK, Fayers PM, Grimshaw JM. Determinants of the intracluster correlation coefficient in cluster randomized trials: the case of implementation research. Clin Trials 2005;2:99-107. 
33. Pagel C, Prost A, Lewycka S, et al. Intracluster correlation coefficients and coefficients of variation for perinatal outcomes from five cluster-randomised controlled trials in low and middleincome countries: results and methodological implications. Trials 2011;12:151.
34. Every Woman Every Child. The Global Strategy for Women's, Children's and Adolescents' Health(2016-2030) : Sustainable Development Goals, 2015. 


\title{
Correction: scaling up quality improvement intervention for perinatal care in Nepal (NePeriQIP); study protocol of a cluster randomised trial
}

KC A, Bergström A, Chaulagain D, et al. Scaling up quality improvement intervention for perinatal care in Nepal (NePeriQIP); study protocol of a cluster randomised trial. BMJ Glob Health 2017;2:e000497.

The authors want to alert the readers on the updated statement under the Contributors section.

Contributors: AK and MM conceptualised the study and AB, UE, DC, OB and JW contributed to the development of study design. BL, PS, AP, AsP and CS validated feasibility and acceptability of the study design after initial development. AG, HL, PE, EG, LE and CL made additions and changes to data collection development. AK and MM drafted the first draft of the manuscript. All authors read and approved of the final version.

\begin{abstract}
Open access This is an open access article distributed in accordance with the Creative Commons Attribution Non Commercial (CC BY-NC 4.0) license, which permits others to distribute, remix, adapt, build upon this work non-commercially, and license their derivative works on different terms, provided the original work is properly cited, appropriate credit is given, any changes made indicated, and the use is non-commercial. See: http://creativecommons.org/licenses/by-nc/4.0

(C) Author(s) (or their employer(s)) 2019. Re-use permitted under CC BY-NC. No commercial re-use. See rights and permissions. Published by BMJ.
\end{abstract}

BMJ Global Health 2019;4:e000497corr1. doi:10.1136/bmjgh-2017-000497corr1 